\title{
Association between intraoperative cerebral microembolic signals and postoperative neuropsychological deficit: comparison between patients with cardiac valve replacement and patients with coronary artery bypass grafting
}

\author{
Sigrun Kierulf Brækken, Ivar Reinvang, David Russell, Rainer Brucher, Jan L Svennevig
}

\begin{abstract}
Objectives-To examine the association between intraoperative cerebral microembolic signals (MES) and postoperative neuropsychological deficit in patients with valve replacement (VR) and patients with coronary artery bypass grafting (CABG). Methods-Neuropsychological examination (10 tests) was performed 1-2 days before and 2 months after surgery (VR $(n=26)$ and CABG $(n=14))$. The intraoperative number of cerebral MES were monitored from the right middle cerebral artery using transcranial Doppler.

Results-A higher number of cerebral MES were detected in VR patients with neurological deficit (6/26) compared with those without deficit (20/26) (MES median number $2083 v 645, p=0.04)$. No such difference was found in patients with CABG (2/14 $v$ 12/14) (MES median number $50 v 112, \mathrm{p}=0.2)$.

Conclusion-A high number of MES were detected in patients with VR with neuropsychological deficit. In patients with CABG with such a deficit, a low number of MES were detected. This difference in number may be explained by relatively more gaseous emboli in the first and more solid in the second. However, the results in the patients with CABG should be interpreted with caution due to the few patients in this subgroup.

(F Neurol Neurosurg Psychiatry 1998;65:573-576)
\end{abstract}

Keywords: embolism; ultrasound; cardiopulmonary bypass; neuropsychiatry

Neuropsychological deficit is experienced in $30 \%$ of patients examined 2 months after cardiopulmonary bypass (CPB) surgery. ${ }^{12}$ The functions most often affected are concentration, memory, learning, and the speed of mental and visuomotor responses. ${ }^{2}$

The aetiology of neuropsychological dysfunction after CPB is unresolved and is probably multifactorial. Two of the main hypotheses are that the decline in performance is due to microemboli entering the cerebral circulation and/or episodes of reduced cerebral perfusion pressure during surgery.

Recent clinical studies have suggested that neurological $^{3}$ and neuropsychological ${ }^{45}$ performance after CPB surgery is related to the intraoperative number of cerebral microembolic signals (MES). The aim of this study was to determine the association between intraoperative cerebral MES and decline in postoperative neuropsychological performance after valve replacement (VR) and coronary artery bypass grafting (CABG), which are the two most frequent types of CPB surgery.

\section{Material and methods}

PATIENT SELECTION

Patients referred for VR or CABG were included in the study after giving informed consent. The following patient categories were excluded: (1) unstable angina pectoris, (2) age $>70$ years, (3) a history or clinical findings suggesting cerebrovascular or neurological disease, (4) $>50 \%$ stenosis or occlusion of a precerebral carotid or vertebral artery or major intracranial artery assessed by Doppler examination, (5) metabolic or immunological disease, and (6) non-fluency in the Norwegian language.

\section{Valve replacement (VR) group}

Twenty seven patients, 21 men and six women, received mechanical bileaflet valves (Carbomedics).

Coronary artery bypass grafting (CABG) group Fourteen men, of whom two had two and 12 had three vessel disease.

\section{CARDIOPULMONARY BYPASS}

The alphastat strategy was applied during bypass, various membrane oxygenators, and non-pulsative Gambro roller pumps, and moderate hypothermia $\left(32^{\circ} \mathrm{C}\right)$ were used in all patients. 
Table 1 Neuropsychological test bettery and test scores, mean (SD) values in patients examined before (1-2 days) and after (3-5 days and 2 months) CPB surgery with valve replacement (VR) or coronary artery bypass grafting (CABG)

\begin{tabular}{|c|c|c|c|c|c|}
\hline \multirow[b]{2}{*}{ Test } & \multicolumn{2}{|c|}{ Before surgery } & \multirow{2}{*}{$\begin{array}{l}\text { After surgery } \\
\text { (3-5 days) } \\
\operatorname{VR}(n=21)\end{array}$} & \multicolumn{2}{|c|}{ After surgery (2 months) } \\
\hline & $V R(n=27)$ & $C A B G(n=14)$ & & $V R(n=26)$ & $C A B G(n=14)$ \\
\hline \multicolumn{6}{|l|}{ Group 1: } \\
\hline WAIS V & $54(12)$ & $47(15)$ & & & \\
\hline WAIS D & $16(3)$ & $15(3)$ & & & \\
\hline CPM & $30(5)$ & $30(5)$ & & & \\
\hline \multicolumn{6}{|l|}{ Group 2: } \\
\hline 1 CVLT L & $56(12)$ & $48(10) \dagger$ & & $64(10)+\uparrow$ & $58(12)+\uparrow$ \\
\hline CVLT S & $12(3)$ & $10(3)$ & & $13(3) \dagger \uparrow$ & $12(3) \dagger \uparrow$ \\
\hline CVLT L & $13(3)$ & $10(3) \dagger$ & & $14(3)$ & $12(3)+\uparrow$ \\
\hline 2 SDL & $16(8)$ & $17(5)$ & $12(9)$ & $18(6)$ & $17(7)$ \\
\hline 3 WMS & $11(4)$ & $9(3)$ & & $12(4)$ & $11(4)^{\star} \uparrow$ \\
\hline \multicolumn{6}{|l|}{ Group 3: } \\
\hline 4 Trail A & $35(18)$ & $38(11)$ & & $34(16)$ & $35(12)$ \\
\hline 5 Trail B & $85(38)$ & $94(27)$ & & $86(47)$ & $97(45)$ \\
\hline LC & $70(14)$ & $81(14)^{\star}$ & $82(18)+\downarrow$ & $74(18)$ & $80(13)$ \\
\hline 7 DS & $44(14)$ & $36(9)$ & $34(10)+\downarrow$ & $46(14)$ & $41(11) \dagger \uparrow$ \\
\hline 8 CRT & $202(35)$ & $207(39) \dagger$ & & $214(58)$ & $204(33)$ \\
\hline \multicolumn{6}{|l|}{ Group 4: } \\
\hline 9 FAS & $37(8)$ & $35(10)$ & $40(10)$ & $39(10)$ & $36(11)$ \\
\hline 10 Peg D & $71(13)$ & $81(15)^{\star}$ & $93(27) \dagger \downarrow$ & $71(12)$ & 75 (11) \\
\hline Peg. ND & 79 (19) & $85(14)$ & $109(47)+\downarrow$ & $76(14)$ & $82(13)$ \\
\hline \multicolumn{6}{|l|}{ Group 5: } \\
\hline Trait & $35(8)$ & $37(9)$ & & $34(7)$ & $35(11)$ \\
\hline State & $35(8)$ & $34(12)$ & $36(7)$ & $30(9) \dagger \uparrow$ & $32(11)$ \\
\hline
\end{tabular}

* $\mathrm{p} \leqslant 0.05 ;+\mathrm{p} \leqslant 0.01 ; \mathrm{D}=$ dominant hand; $\mathrm{ND}=$ non-dominant hand; group $1=$ premorbid function; group 2=memory; group $3=$ attention, concentration, and psychomotor speed; group $4=$ others; group $5=$ anxiety tests used; WAIS V=WAIS vocabulary; WAIS $\mathrm{D}=$ WAIS picture completion; $\mathrm{CPM}=$ raven coloured progressive matrices; CVLT=California auditory verbal learning; $S D L=$ serial digit learning (8 digits); WMS=Wechsler memory scale drawing; Trail A and Trail $\mathrm{B}=$ the trail making tests part A and part B; LC=letter cancellation task; DS=WAIS digit symbol; CRT $=$ computerised reaction time test; FAS=controlled oral word association test; Peg=grooved pegboard test. $\downarrow=$ Deteriorated and $\uparrow=$ improved performance compared with the preoperative examination. ${ }^{\star}$ and † in CABG patients' column preoperatively indicates lower performance than the VR patients.

TRANSCRANIAL DOPPLER MONITORING

Doppler examination of the right middle cerebral artery (MCA) was performed from five minutes before cannulation of the aorta until after decannulation, when closure of the sternum was initiated. The examination was performed with a TC 2000S (Nicolet/EME) (386 computer) with 128 point colour coded FFT. The sample volume was fixed at $10 \mathrm{~mm}$ and the sweep was kept constant and corresponded with a time window overlap in the range between $50 \%$ and $65 \%$. A $2 \mathrm{MHz}$ flat monitoring probe was used for monitoring the MCA at a depth of 48-56 mm. All Doppler findings were recorded on videotape (Panasonic AG 7355, Matsushita Electrical Industrial) for off line analysis.

MICROEMBOLIC SIGNALS

The MES were counted both by an observer using the identification criteria of the consensus committee ${ }^{6}$ and automatically using a specially designed software (RB 11). ${ }^{7}$ The observer assessed the signals according to their sound, duration, appearance on the monitor, and the presence or absence of obvious artefact causes during the registration. When the automatic system and the observer disagreed, the observer's assessment was used for further analysis. We have previously found a $94 \%$ sensitivity between software and observer. During periods with a very high number of MES, observer counting was impossible and only the RB 11 software was used. This program identifies MES as an enhanced power ratio of at least $3 \mathrm{~dB}$ and duration $<300 \mathrm{~ms}$. The number of MES are presented as the total median number of MES per operation.
NEUROPSYCHOLOGICAL EXAMINATION

All patients underwent a neuropsychological examination 1-2 days before, and 2 months after surgery. The neuropsychological tests were modelled on the battery developed by Newman ${ }^{2}$ (table 1). Five of the tests were used for a limited screening in patients with VR during the first week after surgery (table 1). Except for the brief postoperative assessment, administration of the tests was performed blinded to the type of surgery and the number of MES. All neuropsychological tests were scored blinded for this information.

\section{Neuropsychological deficit}

Neuropsychological deficit was defined according to the criteria proposed by Newman. ${ }^{2}$ A deterioration $\geqslant 1$ SD unit for all preoperative scores in all patients in a test was defined as a neuropsychological impairment in one test. For an impairment to indicate neuropsychological deficit, it had to occur in two or more different tests.

\section{CLINICAL NEUROLOGICAL EXAMINATION}

A clinical neurological examination was carried out at each neuropsychological examination.

\section{STATISTICS}

The statistical analysis was based both on two independent groups of patients (VR $(n=26)$ and CABG $(n=14))$ and two outcome variables, neuropsychological deficit 3-5 days and 2 months after surgery. The neuropsychological outcome variables were dichotomised. Mann-Whitney non-parametric test was used to compare groups of patients and the paired $t$ test when matched data were analysed. The 
Table 2 Association between neuropsychological deficit and preoperative and intraoperative variables (values are median (range))

\begin{tabular}{|c|c|c|c|}
\hline \multirow[b]{3}{*}{ Variables } & \multirow{2}{*}{$\begin{array}{l}\text { After surgery ( } 3-5 \text { days) } \\
\operatorname{VR}(n=21)\end{array}$} & \multicolumn{2}{|c|}{ After surgery (2 months) } \\
\hline & & $C A B G(n=14)$ & $V R(n=26)$ \\
\hline & $\begin{array}{l}\text { deficit }+(n=14) \\
\text { deficit }-(n=7)\end{array}$ & $\begin{array}{l}\text { deficit }+(n=2) \\
\text { deficit }-(n=12)\end{array}$ & $\begin{array}{l}\text { deficit }+(n=6) \\
\text { deficit }-(n=20)\end{array}$ \\
\hline \multirow[t]{2}{*}{ Age (y) } & $59.5(40-69) \dagger$ & $64(63-64)$ & $60(43-67)$ \\
\hline & $44(38-70)$ & $57(39-70)$ & $55(38-69)$ \\
\hline \multirow[t]{2}{*}{ Education (y) } & $11.5(7-21)$ & $7(7-7)^{\star}$ & $11.5(7-21)$ \\
\hline & $11(9-16)$ & $11(7-22)$ & $12(7-21)$ \\
\hline \multirow[t]{2}{*}{ NYHA functional class } & $3(1-4)$ & $3(3-3)$ & $3(2-4)$ \\
\hline & $3(2-3)$ & $3(2-3)$ & $3(2-4)$ \\
\hline \multirow[t]{2}{*}{ Ejection fraction (\%) } & $73(48-88)$ & $59(47-71)$ & $59(52-88)$ \\
\hline & $63(50-75)$ & $51(25-82)$ & $67(48-88)$ \\
\hline \multirow[t]{2}{*}{ Cross clamping time (min) } & $55(29-95)$ & $46(34-58)$ & $57(29-112)$ \\
\hline & $71(45-93)$ & $36(19-63)$ & $56(42-93)$ \\
\hline \multirow{2}{*}{ Minimal nasopharyngeal temperature $\left({ }^{\circ} \mathrm{C}\right)$} & $27.6(23.2-31.1)$ & $27.0(25.4-28.5)$ & $27.0(25.8-29.1)$ \\
\hline & $28.8(26.3-29.8)$ & $28.4(25.0-31.7)$ & $28.3(23.2-31.1)$ \\
\hline \multirow{2}{*}{ Cardiopulmonary bypass time (min) } & $93(46-145)$ & $80(68-91)$ & $91(46-161)$ \\
\hline & $103(72-132)$ & $72(41-116)$ & $93(68-132)$ \\
\hline \multirow[t]{2}{*}{ Cerebral MES (n) } & $1155(151-3074)^{\star}$ & $50(32-67)$ & $2083(251-4541)^{\star}$ \\
\hline & $226(78-1870)$ & $112(19-799)$ & $645(78-3074)$ \\
\hline \multirow[t]{2}{*}{ Respirator time $(\mathrm{h})$} & $6(1-18)$ & $6.5(5-8)$ & $9(5-19)^{\star}$ \\
\hline & $6(2-13)$ & $6(2-12)$ & $6(1-13)$ \\
\hline
\end{tabular}

${ }^{\star} \mathrm{p} \leqslant 0.05 ; \dagger \mathrm{p} \leqslant 0.01$. Cross clamping time $=$ the period the aorta was cross clamped and the patient was supported by the heart-lung machine; cardiopulmonary bypass time $=$ the total period the patient was connected to the heart-lung machine; NYHA=NewYork Heart Association.

following variables were analysed to detect a possible association with neurological deficit: patient's age, duration of education, preoperative New York Heart Association (NYHA) functional class, left ventricular ejection fraction (EF), aortic cross clamping time, minimal nasopharyngeal temperature during $\mathrm{CPB}$, $\mathrm{CPB}$ time, the number of MES, and respirator time. Statistical analysis was performed with the Epi Info version 5.01A (Center of Disease Control, Atlanta, USA).

\section{Results}

There were no significant differences between the two groups for age, years of education, minimal nasopharyngeal temperature, or NYHA class. Ejection fraction was lower in the CABG group and the cross clamping and cardiopulmonary bypass times were longer in the patients with VR $(\mathrm{p}<0.05)$.

One patient in the VR group died 6 weeks postoperatively due to cardiogenic shock after dissection of an aortic aneurysm and did not therefore complete the postoperative evaluation.

\section{NEUROLOGICAL ASSESSMENT}

The following neurological abnormalitiesmental confusion, mild hemiparesis, unilaterally increased tendon reflexes, and primitive reflexes-were found in six $(29 \%)$ of the patients at the early and in seven $(18 \%)$ of the patients at the late postoperative examination (CABG $n=4, V R n=3$ ). There was no association between new clinical neurological abnormalities and cerebral MES $(\mathrm{p}=0.8, \mathrm{p}=0.9$ and $\mathrm{p}=0.09$ respectively).

\section{NEUROPSYCHOLOGICAL ASSESSMENTS}

Early postoperative assessment

Five patients with VR refused to participate. Neuropsychological deficit was detected in 14 $(67 \%)$ (median two tests, range 2-5) of the 21 VR patients who completed the examination 3-5 days after surgery. These 14 patients had a lower preoperative score in WAIS picture completion (WAIS D) (mean 15 (SD 3) $v$ mean 18 (SD 2), $\mathrm{p}=0.03$ ), but not in WAIS vocabulary (WAIS V) and Raven coloured progressive matrices (Raven CPM) compared with those without deficit. A higher number of MES were found in the 14 patients with deficit (median 1155), compared with those without (median 226) $(\mathrm{p}=0.03)($ table 2$)$.

\section{Late postoperative assessment}

Valve replacement group-Neuropsychological deficit was found in six of the 26 patients $(23 \%)$ (median two tests, range 2-3) on examination 2 months postoperatively. A lower preoperative score in Raven CPM (27 (SD 4) v 31 (SD 4), $\mathrm{p}=0.04$ ), but not in WAIS $\mathrm{D}$ or WAIS $\mathrm{V}$ was found in those with deficit compared with those without. A higher number of MES were found in the six patients with deficit (median 2083, range 251-4541) compared with the 20 patients without such deficit (median 645, range 78-3074, $\mathrm{p}=0.04$, table 2).

$C A B G$ group - Neuropsychological deficit was found in two of the 14 patients $(14 \%)$ on examination 2 months after surgery. These two patients had a lower median number of MES (median 50, range 32-67) compared with those without deficit (median 112, range 19-799, $\mathrm{p}=0.2$, table 2). No difference in preoperative scores in WAIS D, WAIS V, or Raven CPM was found between the patients with and those without neuropsychological deficit.

ASSOCIATION BETWEEN CLINICAL NEUROLOGICAL DEFICIT AND NEUROPSYCHOLOGICAL DEFICIT At the early examination, four (19\%) patients had both neurological deficit and neuropsychological deficit. Two months after surgery none of the patients had both. 


\section{Discussion}

VR and CABG differed with regard to the number of intraoperative cerebral MES and their time of occurrence. ${ }^{8}$ In the present study VR and CABG patients also differed with regard to the association between cerebral MES and neuropsychological deficit. A higher number of MES was detected in VR patients with neuropsychological deficit. During VR, most MES were detected as the heart regained effective ejection. ${ }^{8}$ These MES are assumed to be reflections from air bubbles leaving the heart and entering the cerebral circulation. This is supported by a transoesophageal echocardiography study which showed retained air in the left side of the heart or the pulmonary veins before effective ejection. ${ }^{9}$

The highest number of MES during CABG were found during clamping and declamping and the period when the aorta was cross clamped. ${ }^{8} 10$ Two previous studies ${ }^{45}$ performed in patients with CABG reported that neuropsychological deficit 8 weeks after surgery was associated with a higher number of intraoperative cerebral MES. However, we found a low number of MES in patients with CABG and neuropsychological deficit. This result should be interpreted with caution, due to the few patients in this subgroup. However, CABG patients may have a relatively higher number of solid emboli than VR patients. This is supported by a recent study where the number of emboli detected by TCD and transeosophagal echocardiography arising with clamp placement and release during CABG, was correlated with the severity of aortic atherosclerosis. ${ }^{10}$

As previously reported, ${ }^{11}$ we found a relation between advanced age and poorer neuropsychological outcome. The mechanism by which age increases neuropsychological deficit is unknown. Advanced age was not found to have any effect on the cerebral blood flow $(\mathrm{CBF})$ response to changes in mean arterial pressure or on the coupling between $\mathrm{CBF}$ and cerebral metabolic rate for oxygen. ${ }^{12}$ Increasing atherosclerosis with asymptomatic cerebrovascular disease is the most commonly proposed explanation for the postoperative decline in neuropsychological performance with advanc- ing age. We found some indication that patients with postoperative neuropsychological impairment had lower preoperative function. This association was only found for one of three preoperative measures, and it was not consistent for the short term and long term outcome. It is reasonable to assume that patients with lower premorbid intellectual functioning may have a higher risk for postoperative cognitive impairment. However, this could not be shown in our study as patients were selected on strict medical criteria and before surgery were intellectually normal.

In conclusion, this study has shown a positive association between the intraoperative number of MES and postoperative deficit in patients with VR but not in patients with CABG.

The study was supported by The Norwegian Council on Cardiovascular Diseases.

1 Shaw PJ, Bates D, Cartlidge NEF, et al. Neurologic and neuropsychological morbidity following major surgery: Comparison of coronary artery bypass and peripheral vascular surgery. Stroke 1987;18:700-7.

2 Newman S. The incidence and nature of neuropsychological morbidity following cardiac surgery. Perfusion 1989;4: 93-100.

3 Clark RE, Brillman J, Davis DA, et al. Microemboli during coronary artery bypass grafting. $\mathcal{F}$ Thorac Cardiovasc Surg 1995;109:249-58.

4 Pugsley W, Klinger L, Paschalis C, et al. The impact of microemboli during cardiopulmonary bypass on neuropsychological functioning. Stroke 1994;25:1393-9.

5 Stump DA, Newman SP. Embolus detection during cardiopulmonary bypass. In: Tegeler $\mathrm{CH}$, eds. Neurosonology. St Louis: Mosby;1996:252-5.

6 Consensus committee of the ninth international cerebral hemodynamic symposium. Basic identification criteria of Doppler microembolic signals. Stroke 1995;26:1123.

7 Van Zuilen EV, Mess WH, Jansen C, et al. Automatic embolus detection compared with human experts. Stroke lus detection com

8 Brækken SK, Russell D, Brucher R, et al. Cerebral Brækken SK, Russell D, Brucher R, et al. Cerebral
microembolic signals during cardiopulmonary bypass surgery. Frequency, time of occurrence, and the association with patient and surgical characteristics. Stroke 1997;28: 1988-92.

9 Orihashi K, Matsuura Y, Hamanaka Y, et al. Retained intracardiac air in open heart operations examined by transesophageal echocardiography. Ann Thorac Surg 1993; 55:1467-71

10 Barbut D, Hinton RB, Szatrowski TP, et al. Cerebral emboli detected during bypass surgery are associated with clamp removal. Stroke 1994;25:2398-402.

11 Sotaniemi KA, Juolasmaa A, Hokkanen TE. Neuropsychologic outcome. A Doppler ultrasound study after openheart surgery. Arch Neurol 1981;38:2-8.

12 Newman MF, Croughwell ND, Blumenthal JA, et al. Effect of aging on cerebral autoregulation during cardiopulmonary bypass. Association with postoperative cognitive dysfunction. Circulation 1994;90:II-234-9. 